\title{
К вопросу о динамических коэффициентах в задачах о сейсмических воздействиях
}

\author{
Е.Н.Курбацкий, РУТ (МИИТ), Москва \\ Г.Э.Мазур, Институт Гипростроймост, Москва \\ В.Л.Мондрус, МГСУ, Москва
}

В первом номере журнала «Аcademia. Архитектура и строительство» в 2019 году была опубликована статья «Динамические коэффициенты или спектры реакций (ответов) сооружений на сейсмические воздействия?» [1]. В статье отмечалось, что в нормах РФ для оценки сейсмических воздействий продолжает использоваться устаревшее (на наш взгляд) понятие «коэффициент динамичности».

Но ни в одном из Сводов правил РФ по расчёту на сейсмические воздействия (СП 14.13330.2018, СП 268.1325800.2016 и др.) нет определения этого понятия. Авторы проекта СП 14.13330.2019 наконец попытались восполнить этот пробел и дать определение коэффициента динамичности через статическое перемещение при землетрясении.

Мы считаем, что такое определение не имеет физического смысла - для сейсмического воздействия невозможно корректно определить статическое воздействие, которое надо умножать на коэффициент динамичности. Оно ничего не определяет и, по всей видимости, введено в текст только ради формального наполнения соответствующего раздела. Наличие в нормативных документах подобных бессмысленных понятий препятствует нормальной работе по совершенствованию нормативной базы и может не только увести в сторону от понимания явления, но и привести к неправильным решениям.

Предлагаем вообще исключить коэффициенты динамичности из нормативных документов РФ, касающихся сейсмических воздействий, и заменить их спектрами реакций (ответов). Обоснованию этого предложения и будет посвящена эта статья.

Концепция спектров ответов признана во всем мире, в том числе и в России. Известно, что количество стран, в нормативных документах которых используется эта концепция, - более пятидесяти.

После публикации статьи «Динамические коэффициенты или спектры реакций (ответов) сооружений на сейсмические воздействия?» мы получили большое количество отзывов как от проектировщиков, так и от известных учёных, которых заинтересовала поднятая нами тема. Поэтому мы считаем необходимым продолжить её обсуждение.

Ключевые слова: сейсмические воздействия, динамические коэффициенты, спектры ответов, зарубежные стандарты, российские нормы.

\section{On the Issue of Dynamic Coefficients in Seismic Impact Problems}

E.N.Kurbatskiy, RUT (MIIT), Moscow

G.E.Mazur, Giprostroymost Institute, Moscow

V.L.Mondrus, MGSU, Moscow

In the first issue of ACADEMIA journal in 2019, an article "Dynamic coefficients or spectra of reactions (responses) of structures to seismic effects?" was published. In the article it was noted that the outdated concept of "dynamic coefficient" continues to be used in the Russian Federation Code.

But in none of the of the Russian Federation seismic Code (SP 14.13330.2018, SP 268.1325800.2016, etc.) there is no definition of this concept. The authors of the project of Code 14.13330.2019 tried to fill this gap and to determine the dynamic coefficient through static displacement during an earthquake.

We believe that such a definition does not have physical meaning. For a seismic impact it is impossible to correctly determine the static impact which must be multiplied by the dynamic coefficient. It does not define anything and, most likely, is entered into the text only for the sake of formal filling of the corresponding section. The presence of such meaningless concept in normative documents impedes normal work on improving the regulatory framework and can not only divert one from understanding the phenomenon, but also lead to incorrect decisions.

We propose to exclude "dynamic coefficients" from the Russian codes relating to seismic effects and replace them with response spectra. This article, is devoted to the rationale for this proposal.

The concept of response spectra is recognized throughout the world, including Russia. It is known that the number of countries where this concept is used in the national codes is more than fifty.

After the publication of the article "Dynamic coefficients or spectra of reactions (responses) of structures to seismic effects?" we received many reviews, both from designers and from wellknown scientists who were affected by the topic that we raised. Therefore, we consider it necessary to continue its discussion.

Keywords: seismic effects, dynamic coefficients, response spectra, foreign standards, Russian codes.

1. Ошибочные положения концепции коэффициентов динамичности при расчёте на землетрясения

Как было отмечено в нашей статье [1], ни в одном из Сводов правил РФ по расчёту на сейсмические воздействия 
не даётся ни определений, ни способов построения «коэффициентов динамичности землетрясений». По-видимому, в ответ на нашу (и не только нашу) критику сейсмических норм в проекте СП 14.13330.2019 появилось определение коэффициента динамичности через «статические перемещения» при землетрясениях, что, по нашему мнению, представляет собой насилие над основными понятиями механики. Приведём это «замечательное» определение.

«Коэффициент динамичности; КД: отношение максимального абсолютного значения динамического перемещения одномерного осциллятора к статическому перемещению. Динамические перемещения - это перемещения осциллятора во времени в ответ на сейсмическое воздействие, заданное в виде акселерограммы. Статическое перемещение - это перемещение осциллятора от действия статической инерционной нагрузки, соответствующей постоянному пиковому ускорению грунта».

Смысл этого запутанного определения можно расшифровать так.

Есть сейсмическое воздействие, заданное двумя способами, - в виде пикового ускорения грунта и в виде акселерограммы (причем пиковое ускорение и акселерограмма могут быть получены различными методами, не связанными между собой; в частности, экстремум акселерограммы может быть и не равен пиковому ускорению!). Для системы с одной степенью свободы (осциллятор в виде массы на пружине или одна из изолированных форм колебаний сложной конструкции) можно получить «неправильные» статические перемещения (относительные!) от условных инерционных сил, равных произведению масс на пиковое ускорение грунта, и «правильные» экстремальные перемещения от воздействия, заданного акселерограммой. Отношение «правильных» перемещений к «неправильным» и есть коэффициент динамичности.

Так определять коэффициент динамичности (делить результат применения коэффициента на первый множитель) бессмысленно. Здесь нет ни физической сути явления, ни требований к моделям. Кроме того, одной акселерограммы для решения задачи недостаточно - необходимо отобрать несколько акселерограмм, получить результаты и творчески их обработать с учетом «мирового опыта», «здравого смысла» или мнения начальства. Несмотря на явную зависимость результата от человеческого фактора, определение всё равно останется верным! С таким же успехом можно пытаться взвесить мешок яблок делением цены мешка на цену одного килограмма яблок...

В многочисленных трудах российских учёных, посвящённых расчёту сооружений на сейсмические воздействия, нам не удалось найти корректное определение коэффициентов динамичности для землетрясений. Единственная известная нам работа, в которой делается попытка обосновать введение коэффициентов динамичности - это статья «0пределение коэффициента динамичности в расчётах на сейсмостойкость» [2]. К сожалению, авторы сделали акцент на второй части своей статьи - на анализе акселерограммы как случайного процесса. Смысл первой части - объяснить читателям с помощью простых формул, что такое «статическая инерционная нагрузка»; откуда берётся динамическое решение авторов не интересует.

Рассмотрим систему с одной степенью свободы. Колебания такой системы при сейсмическом воздействии описывается дифференциальным уравнением:

$$
\ddot{u}_{r}+2 \xi \omega \dot{u}_{r}+\omega^{2} u_{r}=-\ddot{u}_{g},
$$

где: $u_{\mathrm{r}}$ - относительное перемещение массы, $\ddot{u}_{\mathrm{g}}$ - функция ускорений, описывающая колебания основания при землетрясении, $\omega$ и $\xi$ - собственная частота и относительный коэффициент демпфирования системы.

Сейсмическое воздействие представляет собой кинематическое воздействие (колебание основания). Реакция массы (перемещения, скорость и ускорение) на такое воздействие зависит от параметров функции ускорения грунта, собственной частоты и коэффициента демпфирования. Никакие силы при этом на реакцию не влияют.

Отбрасывание первых двух членов в уравнении (1.1) с целью определения не реализуемого при землетрясении статического перемещения, по нашему мнению, не способствует пониманию физического явления. В таком случае перемещения определяются выражением:

$$
\omega^{2} u_{c m}=-\ddot{u}_{\mathrm{g}},
$$

или как представлено в рецензируемой работе:

$$
\omega^{2} u_{c m}=\frac{1}{m} P(t) ; \text { npu } P=\text { const } \rightarrow \omega^{2} u_{c m}=\frac{1}{m} P .
$$

Далее в работе используется понятие «статическая сейсмическая нагрузка» и вводится динамический коэффициент:

$$
\beta=\frac{x_{\partial u t}}{x_{c m}}=\omega^{2} \frac{x_{\partial u m}}{a} .
$$

Затем в работе [2] вводится понятие «спектральное ускорение», которое представляет собой не что иное, как спектр максимальных реакций в ускорениях, и наконец делается вывод, что «КД - это нормированный на величину максимального ускорения спектр ускорений».

Правильно! Но спрашивается, для чего нужно было придумывать и использовать не имеющие физического смысла статические перемещения при землетрясениях?

Несчастный инженер-проектировщик, совсем потеряв нить рассуждений, отправляется искать истину дальше и находит в работе [3] в параграфе 5.4 «0пределение спектров коэффициентов динамичности» указание: «В пространственных задачах динамики и сейсмостойкости возникает необходимость вычислять четыре типа интеграла». Приведём один из них с номером (5.50)

$$
\left\langle\beta_{f_{j} f_{j}}^{2}\left(\Omega_{k}\right)\right\rangle=\Omega_{k}^{4} \int_{0}^{\infty} \frac{G_{f_{j}}(\omega) d \omega}{\left(\Omega_{k}^{2}-\omega^{2}\right)+4 \beta_{k} \omega^{2}}
$$

Далее следует ещё три интеграла такого же типа. Как написано в этом параграфе далее «эти интегралы представляют отношение дисперсий реакций системы при динамических 
и статических воздействиях. Наибольшее распространение получили интегралы (5.50). Как правило, коэффициентами по (5.50) ограничиваются все рекомендации по расчёту сооружений на сейсмические воздействия».

Что же на самом деле представляют собой динамические коэффициенты, рекомендуемые для расчёта на сейсмостойкость в российских СП? Постараемся ответить на этот вопрос.

В соответствии с рекомендациями при определении сейсмической нагрузки, под которой понимаются инерционные силы, массы умножаются на ускорение (коэффициент А), соответствующее расчётной сейсмичности, и коэффициент динамичности, который задаётся в долях ускорения. Таким образом, коэффициент динамичности, умноженный на ускорение, представляет собой ни что иное, как спектр ответов в ускорениях.

Приведём ещё один аргумент в пользу использования спектров ответов. Ни в сводах правил, ни в монографиях российских авторов по расчёту сооружений на сейсмостойкость нет не только правильных и корректных определений коэффициентов динамичности для землетрясений, но и способов их расчёта. Как были получены формулы и кривые коэффициентов динамичности, которые рекомендуются в сводах правил, какие при этом использовались модели нигде не объясняется. Единственное объяснение, которые мы нашли: динамические коэффициенты были получены в результате статистической обработки акселерограмм реальных землетрясений. График коэффициентов динамичности $\beta(T)$, предложенный И.Л. Корчинским шестьдесят лет назад, был включён в СН 8-57 «Нормы и правила строительства в сейсмических районах». График был получен в условиях ограниченного в то время количества инструментальных данных. Отметим такой факт: выборки инструментальных записей, используемые для обоснования параметров трёх графиков $\beta(T)$, включённых в СНиП II-7-81, содержали всего 55 акселерограмм, из которых 30 имели максимальные амплитуды от 0,004 до 0,03 и только одна - более $0,2 \mathrm{~g}$ [5]. Но в настоящее время существуют базы инструментальных записей колебаний грунта, описывающие сейсмические воздействия - «European Strong Motion Database», «ISNM», «K-NET», «KIK-NET» и др. Используя эти базы, можно получить информацию о более чем 50 тысячах землетрясений, которые произошли за последние 30 лет в различных точках земного шара, в том числе и на территории РФ.

Считаем, что разработчиками норм в СССР и РФ была допущена серьёзная методическая ошибка, когда спектры ответов свели к динамическим коэффициентам. Поэтому в нормативных документах следует постепенно отказываться от не имеющих физического смысла динамических коэффициентов при землетрясениях и при расчётах на сейсмические воздействия с использованием спектральной теории применять концепцию спектров ответов. При этом термин «динамический коэффициент» можно было бы заменить на «спектр максимальных реакций в ускорениях». Тем более что спектры ответов - более широкое понятие и, кроме спектра реакций в ускорениях, в расчётах (например, при расчёте сейсмоизоляции) удобно использовать спектры максимальных реакций в скоростях и спектры максимальных реакций в перемещениях. Как можно использовать спектры ответов на практике, мы покажем ниже.

2. Достоинства и преимущества концепции спектров ответов по сравнению с концепцией динамических коэффициентов при землетрясениях

Концепция спектров ответов имеет богатую историю. Впервые идея использования спектров максимальных реакций для представления сейсмических воздействий была изложена в 1926 году в статье К.А. Сюэхиро [6; 7]. Его анализатор сейсмических вибраций состоял из 13 различных масс, подвешенных на пружинах. Собственные частоты этих масс лежали в диапазоне от 0,55 до 4,5 Гц.

Во время землетрясений колебания масс записывались на вращающиеся барабаны. На каждой диаграмме выбиралась только одна точка - максимальное перемещение данной массы, которая наносилась на график. По горизонтальной оси этого графика откладывались частоты колебаний масс, а по вертикальной - максимальные значения перемещений масс при данном сейсмическом воздействии. В результате получен спектр ответов в перемещениях.

Такие же результаты можно получить теоретическим путём, используя акселерограмму землетрясения и модели, представленные на рисунке 1.
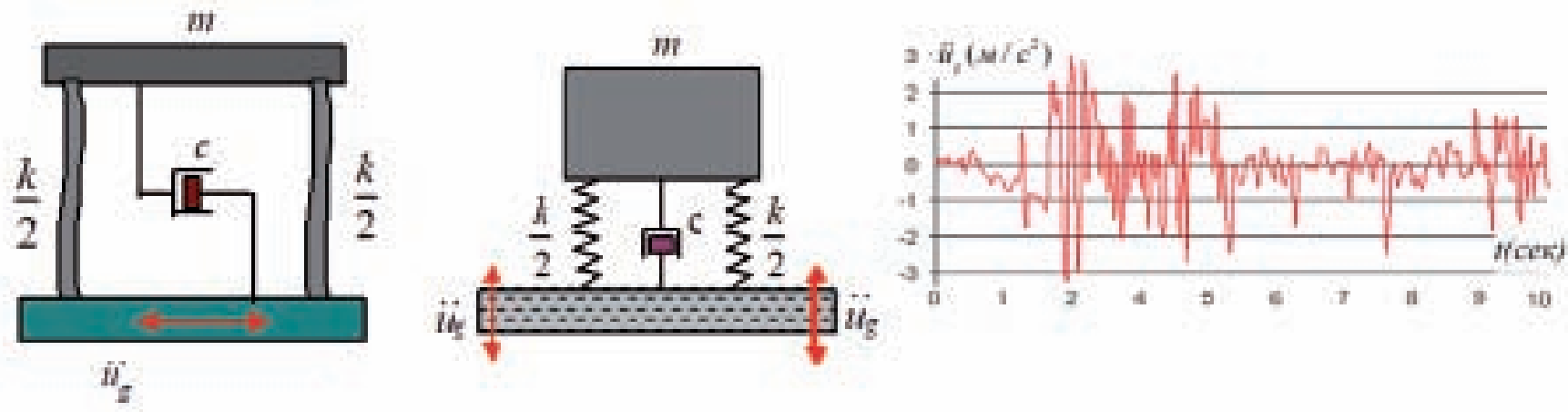

Pис. 1. Модели систем с одной степенью свободы и акселерограмма для построения спектров ответов 
Дифференциальное уравнение движения таких систем имеет вид:

$$
m \ddot{u}_{a}+c \dot{u}_{r}+k u_{r}=0 .
$$

Учитывая, что $\ddot{u}_{\mathrm{a}}=\ddot{u}_{\mathrm{g}}+\ddot{u}_{\mathrm{r}^{\prime}}$ где $\ddot{u}_{\mathrm{a}}-$ абсолютное ускорение, $\ddot{u}_{\mathrm{g}}$ - переносное ускорение и $\ddot{u}_{\mathrm{r}}$ - относительное ускорение, получим:

$$
m \ddot{u}_{r}+c \dot{u}_{r}+k u_{r}=-m \ddot{u}_{g} .
$$

Разделим левую и правую части уравнения (2.2) на массу осциллятора:

$$
\ddot{u}_{r}+2 \xi \omega \dot{u}_{r}+\omega^{2} u_{r}=-\ddot{u}_{g},
$$

где $\omega=\sqrt{\frac{k}{m}} \quad$ - частота собственных колебаний системы без учёта демпфирования, $\quad \xi=\frac{c}{c_{c r}}=\frac{c}{2 m \omega} \quad$ - относительный коэффициент демпфирования (отношение реального демпфирования к критическому).

В правой части уравнения (2.3) есть только один параметр, который характеризует кинематическое возмущение, и нет никаких сейсмических сил.

При сейсмическом воздействии кинематическое возмущение характеризуется функцией горизонтального или вертикального ускорения основания $\ddot{\mathrm{g}}_{\mathrm{g}}$. Отметим очень важный факт [8], который удивляет многих специалистов, воспитанных на псевдостатическом подходе и «динамических коэффициентах», - при землетрясениях наземные части сооружений не подвержены воздействиям никаких внешних сил. Внутренние напряжения и деформации в элементах сооружений создаются исключительно благодаря динамическим реакциям на движение их оснований. Сейсмические воздействия - это кинематические воздействия оснований, а инерционные силы, которые используются в расчётах, - это реакции на кинематическое воздействие.

Для построения спектров ответов на землетрясение в ускорениях, скоростях и перемещениях решается уравнение (2.3) для системы, имеющей собственную частоту $\omega$ и относительный коэффициент демпфирования $\xi$. Из решения выбирается только одно - максимальное значение. Для получения решений используется интеграл Дюамеля и другие численные методы решения дифференциальных уравнений.

Из уравнения (2.1) следует, что при малом затухании

$$
\frac{u_{a}}{u_{g}} \approx-\frac{u_{r}}{m u_{g} / k} \text {. }
$$

Выражение в знаменателе правой части - то самое псевдостатическое смещение осциллятора, которое мы обсуждали выше.

Таким образом, при малом затухании «динамический коэффициент» почти совпадает с нормированным спектром ускорений и является его приближением (а не наоборот!).

Из описания экспериментального метода (опыт Сюэхиро) и теоретического определения максимальных значений ускорений, скоростей и перемещений (уравнение 2.3) следует корректное физически ясное определение спектров максимальных реакций.

\section{1. Простое и физически корректное определение спек-} тров ответов

«Спектр ответов - график максимальных реакций: перемещений, скоростей, ускорений или других максимальных параметров совокупности осцилляторов (систем с одной степенью свободы) на заданное воздействие. Ординаты спектра ответов - максимальные значения реакций осцилляторов на заданное воздействие, абсцисса спектра - собственные частоты осцилляторов или периоды собственных колебаний» [7-9].

Под заданным воздействием при построении спектров максимальных реакций понимается расчётная акселерограмма землетрясения или набор акселерограмм.

\section{2. Логичная и простая методика построения спектров ответов}

Для построения спектров ответов на землетрясение решается уравнение (2.3) для системы, имеющей собственную частоту $\omega$ и относительный коэффициент демпфирования $\xi$. Из решения выбирается только одно максимальное значение. Причём в зависимости от того, какой спектр ответов строится, определяются максимальные ускорения, скорости или перемещения. Затем меняется собственная частота, и процесс повторяется. Для построения спектров ответов рассматривается весь возможный диапазон собственных частот сооружений и относительных коэффициентов демпфирования. Кроме того учитывается, что энергия колебаний грунта переносится гармониками, лежащими в диапазоне до некоторой предельной частоты $f_{\max }$ поэтому область собственных частот сооружений, для которых определяются максимальные перемещения, скорости и ускорения, должна быть менее этой частоты. Как следует из анализа реальных землетрясений, $f_{\max } \leq 33$ Гц. На рисунке 2 представлены спектры ответов в перемещениях, скоростях и ускорениях при воздействии Нортридж (Калифорния, 1994).

2.3. Простая методика построения спектров ответов для систем с разными коэффициентами демпфирования

Для учёта демпфирующих свойств конструкций из различных строительных материалов необходимо построить семейство спектральных кривых с различными относительными коэффициентами демпфирования:

- для конструкций из стали коэффициент демпфирования равен $\xi=0,02$, если уровень напряжений меньше, чем $1 / 2$ от напряжений предела текучести;

- для бетона коэффициент демпфирования зависит от уровня напряжений и находится в пределах от $\xi=0,02$ до $\xi=0,04$;

- для резинометаллических опор, которые используются при сейсмоизоляции, этот коэффициент может меняться в широких пределах от $\xi=0,06$ до $\xi=0,12$.

На рисунке 2 представлены графики спектров максимальных перемещений, скоростей и ускорений при воздействии землетрясения Нортридж для систем с различными коэффициентами демпфирования: $\xi=0,025 ; \xi=0,05 ; \xi=0,10$ и $\xi=0,20$. 

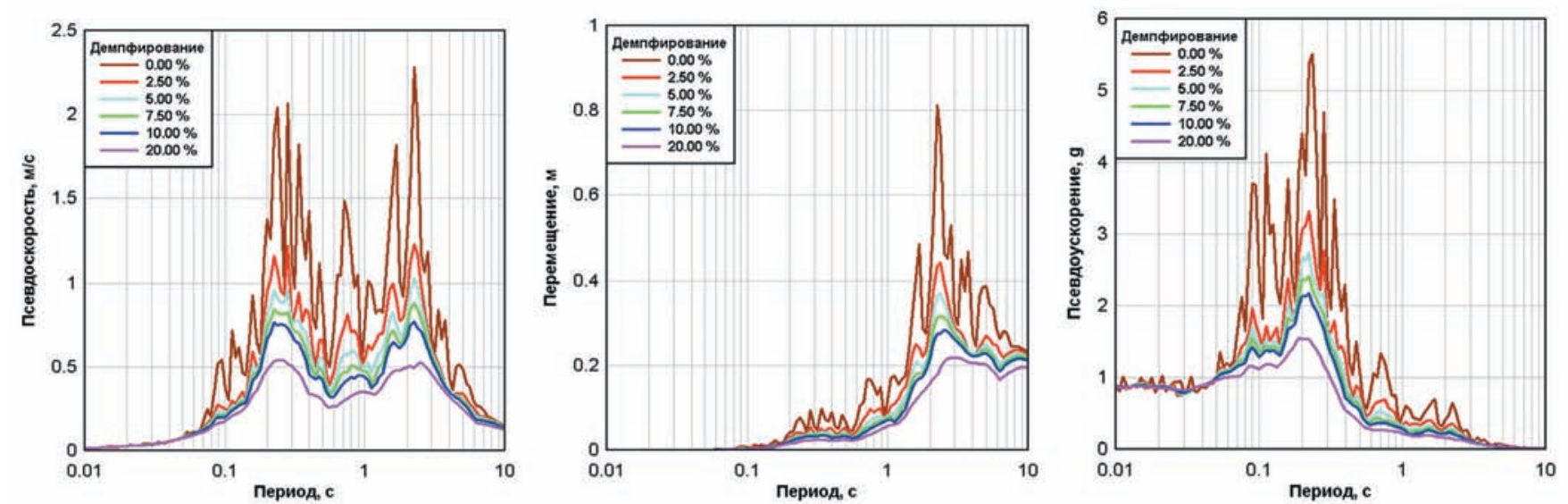

Pис. 2. Спектры ответов в перемещениях, скоростях и ускорениях для систем сразличными коэффициентами демпфирования

\section{4. Построение огибающих спектров ответов по набору акселерограмм землетрясений}

Введение понятий псевдоспектров скоростей $S_{p v}(\omega, \xi)$ и псевдоспектров ускорений $S_{p a}(\omega, \xi)$, справедливых для систем с малыми коэффициентами демпфирования $0<\xi<0,2$, к которым можно отнести большинство строительных конструкций, позволяет получить информацию для построения расчётных спектров ответов. Между псевдоспектрами справедливы соотношения:

$$
S_{p v}(\omega, \xi)=\omega S_{d}(\omega, \xi)=\frac{S_{p a}(\omega, \xi)}{\omega} .
$$

Так как полученный таким образом спектр скоростей не является точным, его называют «псевдоспектром». Использование псевдоспектров позволяет представлять спектры ответов в трёхординатных логарифмических координатах. Учитывая зависимость (2.5), три графика, а именно: график псевдоспектров скоростей, график перемещений и график ускорений, можно совместить в одном.
Представление спектров максимальных перемещений, скоростей и ускорений на одном графике в логарифмических координатах позволило выявить закономерности практически любых сейсмических воздействий. Огибающие спектров большого количества землетрясений определили характерные точки на графиках спектров ответов. Эти точки делят расчётные спектры в области частот или периодов на характерные участки, в которых максимальные реакции - ускорения, скорости и перемещения - принимают постоянные значения.

Именно такие спектры используются для построения спектров в нормативных документах многих стран. Невооружённым глазом видно (рис. 3 левый), какой формы должны быть огибающие спектры. Ньюмарком и Холлом разработана методика построения огибающих спектров (рис. 3 правый). На огибающем спектре видны характерные контрольные точки, которые используются при построении расчётных спектров.
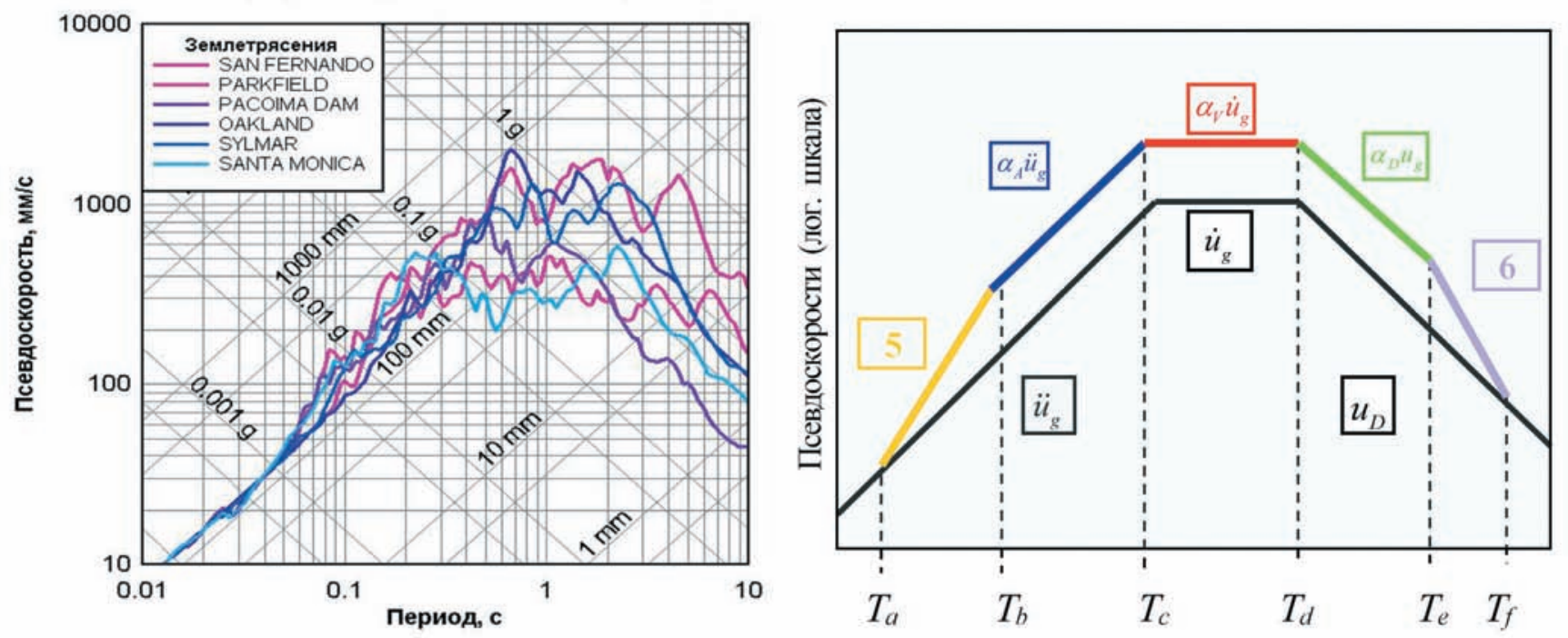

Примечание. Максимальные значения перемещений, скоростей и ускорений грунта представлены линией чёрного цвета.

Рис. 3. Спектры ответов набора акселерограмм различных землетрясений и огибающий спектр Ньюмарка-Холла в логарифмических координатах 
В качестве примера представим спектр международного кодекса IBC. В соответствии с требованиями IBC спектры ответов определяются для шести типов грунта. Спектры откликов имеют разные математические выражения на различных интервалах периодов собственных колебаний. Контрольные точки и значения спектров ответов $S_{\mathrm{D}}$ задаются для каждого типа грунта. Математические выражения спектров откликов в зависимости от периодов колебаний на разных интервалах показаны на рисунке 4.

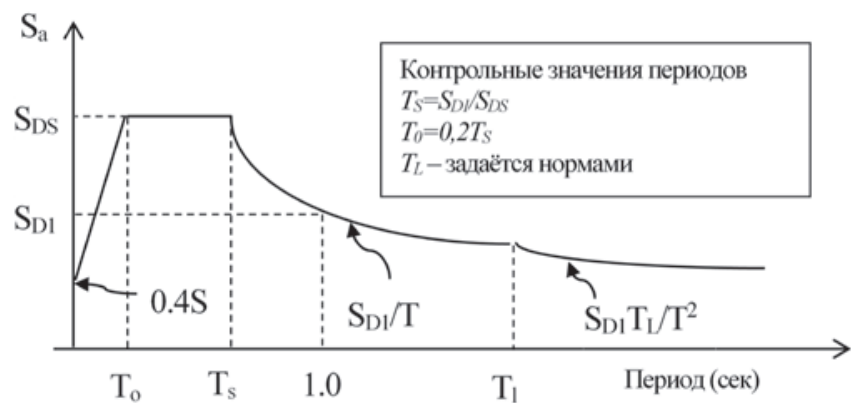

Рис. 4. Спектр откликов (максимальных ускорений) по нормам IBC

Таким образом, в зарубежных нормах, в отличие от российских, для построения спектров ответов существует ясная и понятная методика, представленная в этом пункте и подробно изложенная в монографии [9].

\section{5. Возможность использования спектров ответов для расчёта неупругих систем}

Вполне резонно принять, что правильно рассчитанные и качественно построенные сооружения при воздействии землетрясений слабой и средней интенсивности будут работать в упругой стадии. При сильных, но редких катастрофических сейсмических воздействиях такое предположение практически не реализуемо. Поэтому многими зарубежными нормами, разработанными в последнее время, допускается возможность работы материала конструкций за пределами упругости.

Следует отметить две основные концепции, которые используются при построении спектров максимальных реакций для нелинейных систем: концепцию эквивалентных перемещений и концепцию эквивалентных энергий.

Концепция эквивалентных перемещений является одним из широко используемых методов, учитывающих нелинейное поведение. Концепция позволяет определять коэффициент, уменьшающий силовые воздействия. Концепция эквивалентных перемещений заключается в уменьшении упругих сил, для чего силы умножаются на понижающий коэффициент. Эта концепция является одной из основных в современных расчётах неупругих систем на сейсмические воздействия.

В соответствии с рекомендациями Ньюмарка и Холла при построении спектров ответов для неупругих систем используются спектры ответов упругих систем, которые корректируются множителями, величина которых зависит от частоты (периода).
Для определения спектров ускорения рекомендуется упругие спектры делить на коэффициент $\sqrt{2 \mu-1}$, величина которого определяется из условия эквивалентности энергий для упругой и упругопластической системы.

Подробное изложение концепций эквивалентных энергий и эквивалентных перемещений можно найти в источниках [9-11].

2.6. Возможность построения спектров ответов с учётом соотношения между спектрами ответов в скоростях и амплитудным спектром Фурье ускорений колебаний грунта

Между амплитудными спектрами Фурье и спектрами ответов в скоростях для систем с коэффициентом демпфирования, равным нулю, существует соотношение, которое давно (с 1956 года) известно зарубежным учёным $[13 ; 14]$ :

$$
S_{p v}(\omega, 0)=\left|F\left[\ddot{u}_{g}(t)\right]\right|
$$

Российским учёным, как показывает общение на конференциях, это соотношение между спектрами Фурье и спектрами ответов не известно. Это одно из последствий ошибочной замены определения «спектров ответов» не имеющим физического смысла определением «динамические коэффициенты».

В более позднее время (в 1973 году) американские учёные Ф.Е. Адвэдиа (F.E. Udwadia) и М.Д. Трифунек (M.D. Trifunac) опубликовали статью «Демпфированные спектры Фурье и спектры ответов» [14], в которой для определения взаимосвязи между спектрами Фурье и спектрами ответов для систем с демпфированием использовали новый функционал, который назвали «демпфированный спектр Фурье». Сотрудниками кафедры «Мосты и тоннели» РУТ (МИИТ) подготовлена статья: «Соотношение между спектрами Фурье и спектрами реакций на землетрясения», в которой для определения соотношений между спектрами используется теория функции комплексного переменного (ТФКП) и теория вычетов.

Соотношение между спектрами Фурье и спектрами ответов позволяет более быстро определять спектры ответов, не вычисляя тысячи точек реакций (перемещений, скоростей или ускорений), из которых выбирается только одна - максимум. Соотношения между спектрами Фурье и спектрами ответов удобно использовать для построения поэтажных спектров ответов.

\section{7. Специальные спектры ответов (зависимости} максимальных ускорений от перемещений) для расчёта сейсмоизоляции сооружений

Для анализа параметров сейсмоизолирующих устройств возможно представление спектров реакций и в другом виде: по горизонтальной оси откладываются максимальные перемещения, по вертикальной - максимальные ускорения (рис. 5). На таких графиках периодам собственных колебаний соответствуют прямые радиальные линии, выходящие из начала координат [10]. 
Представление спектров ответов в такой форме позволяет удобно рассчитывать сейсмоизоляцию объектов. На рисунке 5 представлен пример оценки параметров сейсмоизоляции с использованием специального спектра. Точка красного цвета (система без сейсмоизоляции) показывает, что система с относительным демпфированием, равным $2 \%$, и периодом $T=1,0$ сек. при землетрясении будет перемещаться на максимальную величину, равную 0,12 м, с максимальным ускорением $0,5 \mathrm{~g}$.

При увеличении периода до трёх секунд, при том же самом демпфировании, перемещения возрастут почти до 0,4 метра, максимальные ускорения уменьшатся (точка зелёного цвета на рисунке 5). При увеличении демпфирования до $10 \%$ (линия зелёного цвета) уменьшатся и максимальные ускорения, и максимальные перемещения (точка синего цвета). Использование специальных спектров ответов позволяет эффективно и просто определять параметры сейсмоизоляции.

В концепции, основанной на «динамических коэффициентах», провести такой анализ невозможно.

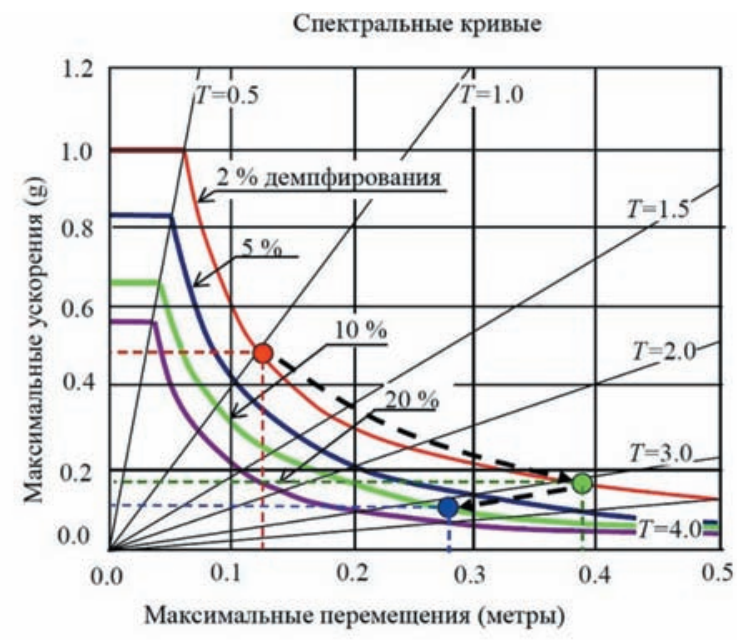

Рис. 5. Специальные спектры ответов - зависимости максимальных ускорений от максимальных перемещений
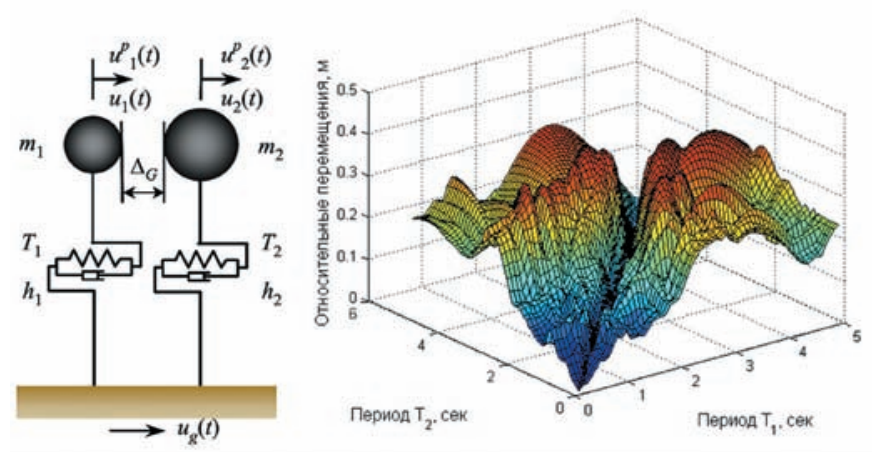

Рис. 6. Модель пролётных строений моста или рядом расположенных сооружений и спектр максимальных относительных перемещений при воздействии землетрясения El Centro. Коэффициенты демпфирования: $\xi_{1}=\xi_{2}=0,05$
2.8. Относительные спектры максимальных перемещений

Спектры максимальных относительных перемещений позволяют определить размеры зон опирания на опорах моста для предотвращения сбросов пролётных строений при землетрясениях. Эти же спектры позволяют оценить возможность соударений при землетрясениях рядом стоящих сооружений.

Можно определить относительные спектры максимальных перемещений с учётом соударений [15].

\section{Заключение}

Представленные определения и методики использования спектров ответов для расчёта сооружений на сейсмические воздействия показывают неоспоримые преимущества этой концепции по сравнению с концепцией динамических коэффициентов.

Концепция, основанная на использовании динамических коэффициентов вместо спектров реакций (ответов), является устаревшей и недостаточной для анализа исходной сейсмической информации в нормах по расчёту сооружений на сейсмостойкость. Положения этой концепции, используемые в нормативных документах, искажают сущность физического явления и могут привести к неправильным решениям.

Можно точно сформулировать, что такое спектры ответов (реакций). Определения «динамических коэффициентов» землетрясений, которые даются в разных источниках, либо неверны, либо неопределённы. И неудивительно - это всего лишь инженерное упрощение одного из видов спектров ответа, и поэтому построить теорию сейсмического расчёта только на основе динамических коэффициентов невозможно.

Следствие, возведённое в абсолют, сделало невозможным понимание причины.

К сожалению, в проекте нового свода правил «Строительство в сейсмических районах» по-прежнему используется ошибочное определение сейсмического воздействия и представлено не имеющее физического смысла определение динамического коэффициента через «статическое перемещение» при землетрясении.

Выражаем твёрдое убеждение, что из нормативных документов следует исключить понятие «динамические коэффициенты», заменив их спектрами реакций (ответов).

\section{Лuтература}

1. Курбацкий, Е.Н. Динамические коэффициенты или спектры реакций (ответов) сооружений на сейсмические воздействия / Е.Н. Курбацкий, В.Л. Мондрус // Academia. Архитектура и строительство. - 2019. - № 1. - С. 107-114.

2. Назаров, Ю.П. Определение коэффициента динамичности в расчётах на сейсмостойкость [Электронный ресурс] / Ю.П. Назаров, Е.В. Позняк // Строительство: наука и образование. - 2015. - № 1. - Режим доступа: http://www. nso-journal.ru/public/journals/1/issues/2015/01/2_Nazarov. pdf (дата обращения 10.10.2019). 
3. Назаров, Ю.П. Расчётные модели сейсмических воздействий / Ю.П. Назаров. - М. : Наука, 2012. - 409 с.

4. Айзенберг, Я.М. Сейсмостойкость оснований // Российская архитектурно-строительная энциклопедия. В 14 томах / Гл.ред. Е.В. Басин. - М. : ВНИИНТПИ, 1995. - Т. 2. - С. 413-414.

5. Поляков, С.В. Зависимость коэффициента динамичности от грунтовых условий площадки строительства / С.В. Поляков, А.М. Кахновский // Анализ последствий землетрясений. - М. : ЦНИИСК им. Кучеренко, 1982. - С. 5-11.

6. Suyehiro, K.A. A seismic vibration analyser and the records obtained therewith / K.A Suyehiro // Bulletin of the Earthquake Research Institute-University of Tokyo. - 1926. - № 1. - P. 59-64.

7. Chopra, Anil K. Elastic response spectrum: a historical note / Chopra Anil K. // Earthquake Engineering and Structural Dynamics. - 2007. - № 36. - P. 3-12.

8. Chopra, Anil K. Dynamics of Structures. Theory and applications to Earthquake Engineering / Chopra Anil K. Pearson Prentice Hall, Person Education Inc. Upper Saddle River, 2007.

9. Hudson, D.E. Response spectrum techniques in engineering seismology / D.E. Hudson // Proceedings of the First World Conference on Earthquake Engineering. - Berkley, CA, 1956.

10. Курбацкий, Е.Н. Спектры Фурье и спектры ответов на землетрясения. Теория и приложения / Е.Н. Курбацкий. - М. : СРОСЭКСПЕРТИЗА, 2018. - 156 с. ISВN 978-5-00077-755-8.

11. NEHRP Recommendation Provision for New Building and Other Structures : Training and Instructional Material // FEMA 451B - June 2007.

12. Hudson, D. E. (1962). Some problems in the application of spectrum techniques to strong-motion earthquake analysis / D.E Hudson // Bull. Seism. Soc. Aт. - № 52. - P. 417-430.

13. Udwadia, F.E. The Fourier Transform, Response Spectra and their relationship through the statistics of oscillator response / F.E. Udwadia, M.D. Trifunac; Earthquake Engineering Research Laboratory, EERL 73-01. - Pasadena : California Institute of Technology, 1973.

14. Udwadia, F.E. Damped Fourier spectrum and response spectra / F.E. Udwadia, M.D. Trifunac // Bulletin of the Seismological Society of America. - 1973. - Vol. 63, No. 5. - P. 1775-1783.

15. Kassawara, P.P. Dynamic Analysis of Structural Systems Excited at Multiple Support Locations / P.P. Kassawara, D.A Peck // Proc., ASCE Specially Conference on Structural Design of Nuclear Plant Facilities. - Chicago, 1973.

\section{References}

1. Kurbatskii E.N., Mondrus V.L. Dinamicheskie koeffitsienty ili spektry reaktsii (otvetov) sooruzhenii na seismicheskie vozdeistviya [Dynamic coefficients or spectra of reactions (responses) of structures to seismic effects]. Akademia. Arkhitektura $i$ stroitel'stvo [Academy. Architecture and construction], 2019, no. 1, pp. 107-114. (In Russ., abstr. in Engl.)
2. Nazarov Yu.P., Poznyak E.V. Opredelenie koeffitsienta dinamichnosti v raschetakh na seismostoikost' [Determination of the coefficient of dynamism in the calculations for earthquake resistance]. Stroitel'stvo: nauka i obrazovanie [Construction: science and education], 2015, no. 1. URL: http// www.nso-journal.ru (Accessed 10.10.2019).

3. Nazarov Yu.P. Raschetnye modeli seismicheskikh vozdeistvii [Design models of seismic effects]. Moscow, Nauka Publ., 2012, 409 p.

4. Aizenberg Ya.M. Seismostoikost' osnovanii [Earthquake resistance]. Rossiiskaya arkhitekturno-stroitel'naya entsiklopediya [Russian architectural and construction encyclopedia], E.V. Basin (ed.). Vol. 2. Moscow, VNIINTPI Publ., 1995, pp. 413-414.

5. Polyakov S.V., Kakhnovskii A.M. Zavisimost' koeffitsienta dinamichnosti ot gruntovykh uslovii ploshchadki stroitel'stva [The dependence of the coefficient of dynamism on the soil conditions of the construction site]. In Analiz posledstvii zemletryasenii [Earthquake impact analysis]. Moscow, TsNIISK im. Kucherenko Publ., 1982, pp. 5-11.

6. Suyehiro K. A seismic vibration analyser and the records obtained therewith. Bulletin of the Earthquake Research. Institute-University of Tokyo 1926, no. 1, pp. 59-64. (In Engl.)

7. Shopra Anil K. Elastic response spectrum: a historical note. Earthquake Engineering and Structural Dynamics, 2007, no. 36, pp. 3-12. (In Engl.)

8. Shopra Anil K. Dynamics of Structures. Theory and applications to Earthquake Engineering. Pearson Prentice Hall, Person Education Inc. Upper Saddle River, 2007.

9. Hudson D.E. Response spectrum techniques in engineering seismology. Proceedings of the First World Conference on Earthquake Engineering. Berkley, CA, 1956. (In Engl.)

10. Kurbatskii E.N. Spektry Fur'e i spektry otvetov na zemletryasenich. Teoriya i prilozheniya [Fourier spectra and response spectra for an earthquake. Theory and Applications]. Moscow, Srosespertiza Publ., 2018, 156 p.

11. NEHRP Recommendation Provision for New Building and Other Structures : Training and Instructional Material. FEMA 451B, June 2007.

12. Hudson D.E. (1962). Some problems in the application of spectrum techniques to strong-motion earthquake analysis. Bull. Seism. Soc. At., 1962, no.52 pp. 417-430.

13. Udwadia, F. E. and M. D. Trifunac (1973a). The Fourier Transform, Response Spectra and their relationship through the statistics of oscillator response. Pasadena, Californialnstitute ofTechnology Publ., 1973.

14. Udwadia, F.E. and M. D. Tifunac. (1973) Damped Fourier spectrum and response spectra. Bulletin of the Seismological Society of America, 1973, vol. 63, no. 5, pp. 1775-1783.

15. Kassawara P.P. and Peck, D.A. Dynamic Analysis of Structural Systems Excited at Multiple Support Locations. Proc., ASCE Specially Conference on Structural Design of Nuclear Plant Facilities, Chicago, III., Dec., 1973. 
Курбацкий Евгений Николаевич (Москва). Доктор технических, академик РАТ. Профессор кафедры мостов и тоннелей ФГАОУ В0 «Российский университет транспорта» (МИИТ). (127994, г. Москва, ул Образцова, д 9, стр. 9. МИИТ). E-mail: dynamic. miit@gmail.com.

Мазур Геннадий Эдуардович. Доктор технических наук. Главный специалист ОАО «Институт Гипростроймост» Эл.почта: mazur.ge@giprosm.ru

Мондрус Владимир Львович (Москва). Доктор технических наук, профессор, член-корреспондент РААСН. Заведующий кафедрой строительной и теоретической механики (СиТМ) ФГБОУ В0 «НИУ МГСУ» (129337, г. Москва, Ярославское шоссе, д. 26. мГСУ). E-mail:mondrus@mail.ru.

Kurbatsky Evgeny Nikolaevich (Moscow). Doctor of Technical Sciences, Academician of the Russian Academy of Transport. Professor of the Department of Bridges and Tunnels at the FGAOU V0 "Russian University of Transport" (MIIT) (9 0braztsova st. building 9, Moscow, 127994. MIIT). E-mail: dynamic.miit@gmail.com.

Mazur Gennady Eduardovich. Doctor of Technical Sciences. Chief specialist of OAO "Institute Giprostroymost" (2 Pavel Korchagin St., Moscow. Giprostroymost). E-mail: mazur.ge@giprosm.ru.

Mondrus Vladimir Lvovich (Moscow). Doctor of Technical Sciences, Professor, Corresponding Member of RAACS. Head of the Department of construction and theoretical mechanics at the Moscow State University of Civil Engineering (26 Yaroslavskoye Highway, Moscow, 129337. MGSU). E-mail:mondrus@mail.ru. 